\title{
Paradigm Shift in Recent Perspectives on Gastric Cancer
}

\author{
Takuji Gotoda \\ Division of Gastroenterology and Hepatology, Department of Medicine, Nihon University School of Medicine, \\ Tokyo, Japan
}

The term "paradigm," which is now widely used, was originally coined in the field of natural sciences as the dominant norm of "perceiving things" in a particular period or field. New findings in a field, however, can create conflict, leading to a needed "paradigm shift." A wellknown example of new evidence creating a paradigm shift is the evolution from the theory of celestial motion to the theory of geocentric motion based on quantitative data. The concept of paradigm proposed during the scientific revolution has been expanded and further refined in recent years, with the term now generally used to describe "thinking outside the box (common sense)" or "overturning of established theories." Herein, we describe a paradigm shift, in its broader sense, to interpret the changes in gastric cancer treatment over time.

Cancer can be generally defined as the continual unregulated proliferation of a group of cells which can invade surrounding tissues as well as metastasize to remote sites in the body. An accepted paradigm about cancer, including gastric cancer which is our focus, is that earlystage cancer, if left untreated, will progress and have a negative impact on prognosis and survival. This "common" understanding of cancer has guided cancer research and treatment. A new finding regarding cancer progression, such as "most early-stage gastric cancers do not affect survival," would be required to create a shift in our accepted paradigm for gastric cancer treatment. However, there are ethical issues in conducting clinical research on humans to change "current accepted practice." It is to address this issue that we propose a paradigm shift in the way we interpret the changes in gastric cancer management over time.

The "present" state of gastric cancer management provides the starting point for our discussion. A recent major reform in the management of gastric cancer has been the introduction of primary prevention by Helicobacter $p y$ lori eradication and population-based endoscopic screening as second prevention. These preventative strategies have greatly improved the outcomes of gastric cancer in Japan [1]. This new nationwide health direction, from treatment to prevention, is a clear paradigm shift in the management of gastric cancer. Moreover, the criteria for endoscopic diagnosis and histological assessments of gastric cancer based on Helicobacter pylori-infected gastric mucosa are also changing [2]. This represents a paradigm shift in our understanding of the association between $\mathrm{He}$ licobacter pylori eradication intervention and gastric cancer. This has led to changes in Helicobacter pylori eradication therapy. Helicobacter pylori eradication therapy should be a simple and well-tolerated regimen, which is cost-effective and generally has good adherence. However, there may be a need for a paradigm shift in Helicobacter pylori eradication therapy considering the increase in clarithromycin-resistant strains and the introduction of new acid-secreting inhibitors [3]. karger@karger.com

www.karger.com/dig (c) 2021 S. Karger AG, Basel

Karger!"
Correspondence to:

Takuji Gotoda, takujigotoda@yahoo.co.jp 
It is worth noting a "past" paradigm shift in the management of early-stage gastric cancer with the advent of the gastroscope. Japan espoused the possibility of curable gastric cancer due to the high incidence of this cancer in this country. This "possibility" led to the development of fiberscopes and high-resolution endoscopes which resulted in the extension of gastrointestinal endoscopy from diagnosis to treatment. It is worth noting that just before the turn of the new century, endoscopic submucosal dissection (ESD) revolutionized the treatment of early-stage gastric cancer [4], with more than half of all gastric cancer cases currently being treated using ESD. This has overturned the "conventional wisdom" of gastric cancer treatment of 20 years ago, which consisted of surgical gastrectomy and lymph node dissection.

Current evidence points to a decrease in the incidence of gastric cancer in the "near future" from a decrease in Helicobacter pylori infection rates and the widespread use of Helicobacter pylori eradication therapy [5]. This change, however, will take time to fully implement; as such, physicians can expect to continue to encounter cases of gastric cancer in routine practice for another 10-15 years. Furthermore, as discussed above, the methods for gastric cancer diagnosis are changing, both at the endoscopic finding and histological level. We are also entering a transitional era with the use of artificial intelligence (AI) in medicine, including the use of AI becoming a powerful tool for the diagnosis of gastric cancer. It is important to also consider that the population at high risk for developing gastric cancer after Helicobacter pylori infection or eradication is naturally getting older. This means that physicians will encounter patients with gastric cancer for whom treatment is not indicated, according to current guidelines [6].
To date, the treatment for gastric cancer has been based on the risk of lymph node metastasis. Yet, it would be more desirable to establish a treatment strategy that is based on a comprehensive evaluation of the patient's physical function, underlying diseases, and nutritional status. Accordingly, it may be time to shift our focus from "care" to "cure" for gastric cancer. Taken together, this Special Issue of Digestion will provide a comprehensive review of the past, present, and future of gastric cancer treatment which will be invaluable to direct the future clinical practice and research in gastric cancer.

\section{Acknowledgment}

I would like to thank Editage (www.editage.com) for English language editing.

\section{Conflict of Interest Statement}

The author has no conflict of interest.

\section{Funding Sources}

The author received no financial support.

\section{Author Contributions}

The sole author prepared the Editorial.

\section{References}

1 Sugano K. Effect of helicobacter pylori eradication on the incidence of gastric cancer: a systematic review and meta-analysis. Gastric Cancer. 2019 May;22(3):435-45.

2 Ono S, Kawada K, Dohi O, Kitamura S, Koike $\mathrm{T}$, Hori S, et al. Linked color imaging focused on neoplasm detection in the upper gastrointestinal tract: a randomized trial. Ann Intern Med. 2021 Jan;174(1):18-24.
3 Suzuki S, Gotoda T, Kusano C, Ikehara H, Ichijima R, Ohyauchi M, et al. Seven-day vonoprazan and low-dose amoxicillin dural therapy as first-line helicobacter pylori treatment: a multicenter randomized trial in Japan. Gut. 2020 Jun;69(6):1019-26.

4 Ono H, Yao K, Fujishiro M, Oda I, Uedo N, Nimura S, et al. Guidelines for endoscopic submucosal dissection and endoscopic mucosal resection for early gastric cancer (second edition). Dig Endosc. 2021 Jan;33(1):4-20.
5 Mizota Y, Yamamoto S. How long should we continue gastric cancer screening? From an epidemiological point of view. Gastric Cancer. 2019 May;22(3):456-62

6 Hatta W, Gotoda T, Oyama T, Kawata N, Takahashi A, Oka S, et al. Is additional surgery always sufficient for preventing recurrence after endoscopic submucosal dissection with curability C-2 for early gastric cancer? Ann Surg Oncol. 2019 Oct;26(11):3636-43. 